Doug Geisler, Eva K. Grebel, and Dante Minniti, eds.

\title{
NGC 3603 - a Local Template for Massive Young Clusters
}

\author{
Bernhard R. Brandl \\ Center for Radio Astronomy and Space Research, Cornell University, \\ Ithaca, NY 14853, USA \\ Wolfgang Brandner \\ European Southern Observatory, Karl-Schwarzschild-Str. 2, D-85748 \\ Garching bei München, Germany \\ Frank Eisenhauer \\ Max-Planck-Institut für Extraterrestrische Physik, Giessenbachstraße, \\ D-85741 Garching, Germany \\ Anthony F. J. Moffat \\ Départment de physique, Université de Montréal, Montréal, Canada \\ Francesco Palla \\ Osservatorio Astrofisico di Arcetri, Largo E.Fermi 5, I-50125 Firenze, \\ Italy
}

Hans Zinnecker

Astrophysikalisches Institut Potsdam, An der Sternwarte 16, D-14482 Potsdam, Germany

\begin{abstract}
We present a study of the star cluster associated with the massive Galactic HII region NGC 3603 based on near-IR broad- and narrowband observations taken with ISAAC/VLT under excellent seeing conditions $\left(\leq 0.4^{\prime \prime}\right)$. We discuss color-color diagrams and address the impact of the high UV flux on the disk evolution of the low-mass stars.
\end{abstract}

\section{Stellar Content and Ionized Gas around NGC 3603}

NGC 3603 is the most massive Galactic H II region and star cluster that can be studied at optical wavelengths. The OB stars contribute more than $2000 M_{\odot}$ to the cluster mass, and provide about 100 times more ionizing power than those of the Trapezium cluster (Moffat, Drissen \& Shara 1994, Kennicutt 1984). At a distance of only $6 \mathrm{kpc}$ it is the perfect laboratory to study starburst-like conditions on a star-by-star basis, and hence the conditions that may lead to the formation and disruption of massive stellar clusters.

Eisenhauer et al. (1998) have studied the stellar IMF in NGC 3603 down to $\sim 1 \mathrm{M}_{\odot}$ using $\mathrm{JHK}$ adaptive optics observations. We have further studied 

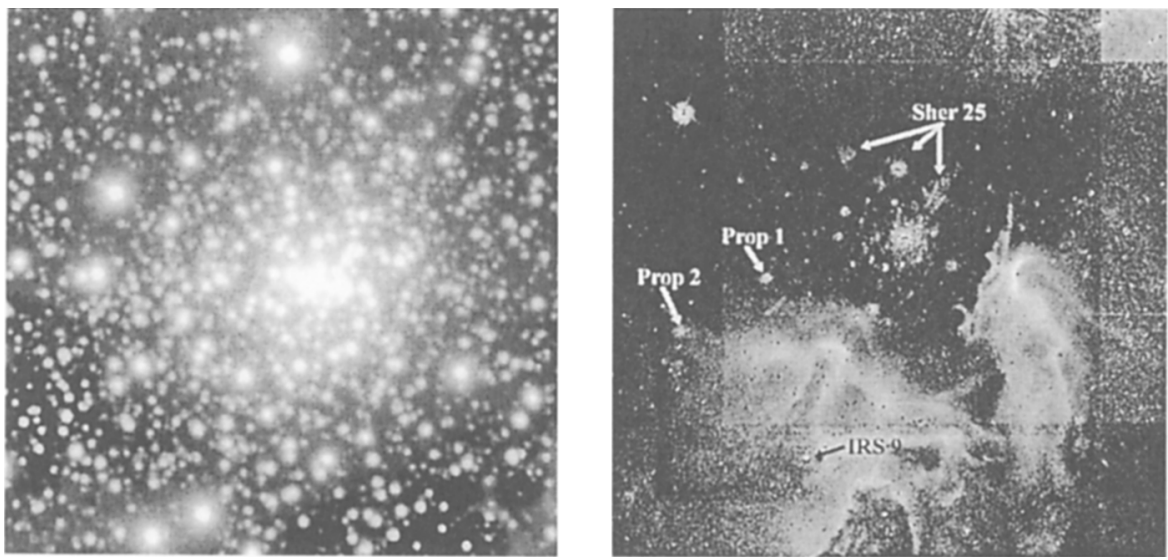

Figure 1. Left: Deep H-band ISAAC/VLT image of the central $50^{\prime \prime} \times$ $50^{\prime \prime}\left(1.55 \times 1.55 \mathrm{pc}^{2}\right)$ of NGC 3603 . Right: 150 s exposure in the Bracket$\gamma$ line of the $2 ! 6 \times 2 ! 6$ around NG $\overline{\mathrm{C}} 3603$; the continuum emission has been subtracted.

the stellar content of NGC 3603 in Brandl et al. (1999) and found that the core region is well populated in low-mass stars down to at least $0.1 \mathrm{M}_{\odot}$ with ages comparable to that of the high mass stars. Figure 1a shows the result of a 45 minute deep H-band image taken with ISAAC at the VLT in April 1999 under excellent seeing conditions $(\leq 0 . " 4)$. The faintest stars visible in this image are about $H=21^{m}$, corresponding to $0.1 \mathrm{M}_{\odot}$ for an age of $1 \mathrm{Myr}$.

We have obtained ISAAC/VLT images in a variety of narrowband filters (Br- $\gamma, \mathrm{H}_{2},[\mathrm{FeII}], \mathrm{HeI}, \mathrm{CO}$-bandhead). Figure $1 \mathrm{~b}$ shows a short exposure in the Bracket- $\gamma$ line with a total integration time of only 150 seconds; the K-band continuum emission has been subtracted. Despite the low signal-to-noise the extended nebular structures and the ionization fronts to the west and southeast of NGC 3603 are clearly visible. The image reveals the ring nebula around the blue supergiant Sher 25 (Brandner et al. 1997) about 18" north of the core and its bipolar outflows to the east and west. The proplyd-like structures, recently discovered by Brandner et al. (2000), are clearly visible. The deeply embedded proto cluster IRS 9 about $1^{\prime}$ south of NGC 3603 becomes almost invisible after continuum subtraction.

Figure 2 shows the color-color-diagram for all stars within the central parsec $\left(r \leq 33^{\prime \prime}\right)$ detected in all three JHK bands on the same pixel. To reduce the contamination from field stars we have statistically subtracted the stellar density in the outer parts of our 3.4 large FOV. The solid line in Fig. 2 indicates the evolution along the (pre-) main sequence for a foreground extinction of $A_{V}=$ $4.5^{m}$. The separation in two "clumps" indicates that only stars more massive than about $\mathrm{A} 5 \mathrm{~V}\left(\sim 2 \mathrm{M}_{\odot}\right)$ have reached the main sequence.

Note that classical T-Tauri or Herbig AeBe stars would fall in a region to the right of the lower dashed line (extinction vector). IR-excess emission from disks and circumstellar matter would make their colors significantly different. Despite the young age, there is no evidence for a significant percentage of young 


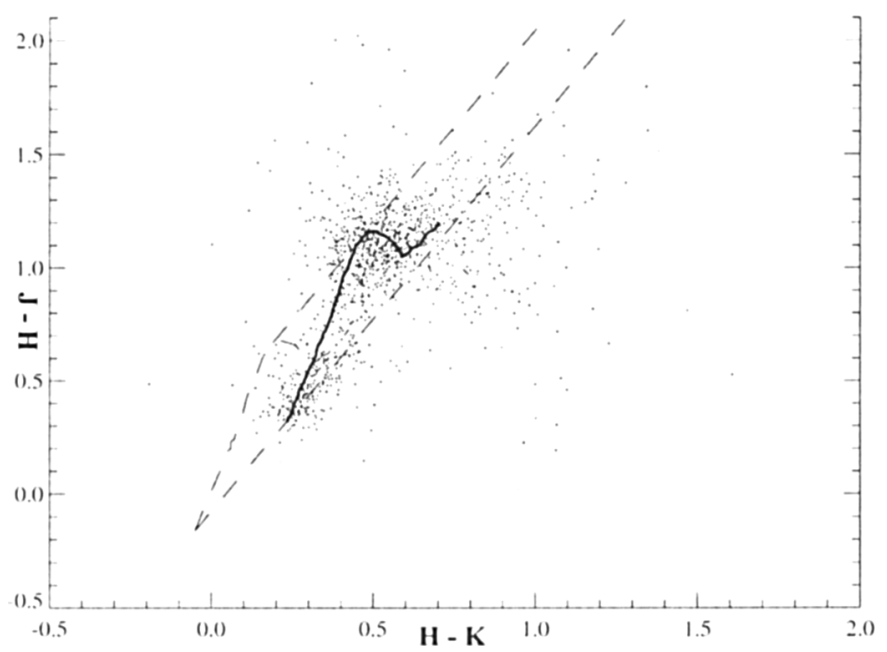

Figure 2. $\quad(\mathrm{J}-\mathrm{H}) /(\mathrm{H}-\mathrm{K})$ color-color diagram for all stars within $r \leq$ $33^{\prime \prime}$, where the field stars have been statistically subtracted. The long dashed lines represent the reddening vectors. The thick line shows the location of (pre-) main sequence stars for $A_{V}=4.5^{m}$.

stars with circumstellar matter within the central parsec, probably because of photoevaporation due to the high UV flux generated by the massive stars. If this result is generally true, no planetary systems should be expected in globular clusters.

Acknowledgments. We'd like to thank Eva Grebel and Doug Geisler for organizing a stimulating meeting in a beautiful location (Pucon).

\section{References}

Brandl, B., Brandner, W., Eisenhauer, F., Moffat, A. F. J., Palla, F., Zinnecker, H. $1999, \mathrm{~A} \& \mathrm{~A}, 352 \mathrm{~L}, 69$

Brandner, W. et al. 1997, ApJ, 489, L153

Brandner, W., Grebel, E. K., Chu, Y.-H., Dottori, H., Brandl, B., Richling, S. Yorke, H. W., Points, S. D., Zinnecker, H. 2000, AJ, 119, 292

Eisenhauer, F., Quirrenbach, A., Zinnecker, H., Genzel, R. 1998, ApJ, 498, 278

Kennicutt, R. C. Jr. 1984, ApJ, 287, 116

Moffat, A. F. J., Drissen, L., Shara, M. M. 1994, ApJ, 436, 183 